\title{
Magnetic forces and localized resonances in electron transfer through quantum rings
}

\author{
M.R. Poniedziałek and B. Szafran \\ Faculty of Physics and Applied Computer Science, AGH University of Science and \\ Technology, al. Mickiewicza 30, 30-059 Kraków, Poland
}

\begin{abstract}
We study the current flow through semiconductor quantum rings. In high magnetic field the current is usually injected to the arm of the ring preferred by classical magnetic forces. However, for narrow magnetic field intervals that appear periodically on the magnetic field scale the current is injected to the other arm of the ring. We indicate that the appearance of the anomalous - non-classical - current circulation results from Fano interference involving localized resonant states. The identification of the Fano interference is based on the comparison of the solution of the scattering problem with the results of the stabilization method. The latter employs the bound-state type calculations and allows to extract both the energy of metastable states localized within the ring and the width of resonances by analysis of the energy spectrum of a finite size system in function of its length. The Fano resonances involving states of anomalous current circulation become extremely narrow on both magnetic field and energy scales. This is consistent with the orientation of the Lorentz force that tends to keep the electron within the ring and thus increases the lifetime of the electron localization within the ring. Absence of periodic Fano resonances in electron transfer probability through a quantum ring containing an elastic scatterer is also explained.
\end{abstract}




\section{Introduction}

The Aharonov-Bohm effect [1] consists in interference of parts of the electron wave function passing through two paths that contain a magnetic flux inside. In the originally considered situation [1] the magnetic field is zero within the region accessible to electrons. The experiments are usually performed in a spatially homogenous magnetic field so the Aharonov-Bohm phase shifts are accompanied by magnetic deflection of the electron trajectories. In particular, measurements performed for the Young double slit experiment in vacuum [2] indicate that the envelope of the interference pattern is shifted as due to the classical Lorentz force. In solids [3] the magnetic deflection of the electron trajectories can influence the results of the interference only provided that the Larmor (cyclotron) radius is comparable to the width of the channels. For metal mesoscopic systems [4] this condition is hardly fulfilled. In semiconductors the magnetic deflection of electron trajectories may be significant even for the width of the channels of the order of several tens of nanometers [5]. Recently, the magnetic deflection was used in the proposal [6] of a solid-state setup for the interaction-free-measurement.

The effects of magnetic forces were studied for the electron injection for semiconductor quantum point contacts [7] and quantum rings [8]. In two-terminal quantum rings [8] the magnetic forces lead to a preferential injection of the electron to one of the arms of the ring (i.e. to the left arm for $B>0$ ). In consequence, the parts of the electron wave function that meet at the exit to the output channel are unequal which reduces the amplitude of Aharonov-Bohm oscillations at high magnetic field $(B)$. In three-terminal quantum rings [8] the magnetic forces besides the reduction of the Aharonov-Bohm oscillation amplitude lead to a distinct imbalance of the transfer probability to the two output terminals. An experiment on magnetic forces in three terminal quantum ring performed only recently [9] confirmed precedent theoretical predictions [8]. The studies of magnetic forces [8] were based on wave packet dynamics. In our previous work [10] we provided a study of magnetic forces in stationary electron flow. The stationary solution generally reproduces the qualitative features of the wave packet dynamics simulations [8]. However, at high magnetic field we found [10] an abrupt reversal of the current circulation and a resulting switch in the transfer probabilities to the two output leads. It was found [10] that the interference conditions leading to the reversed current circulation appear for very narrow $B$ intervals. The physical origin of the anomalous current circulation was not explained. In the present paper we demonstrate that the reversed current circulation is due to Fano interference involving ring-localized states with magnetic dipole moment that is antiparallel to the external magnetic field.

The initial and finite states in the electron scattering problem correspond to delocalized states of the energy continuum. The resonant scattering is qualitatively understood [11] as due to presence of a metastable state localized at the scattering object that after a finite lifetime $\tau$ decays elastically into the delocalized state. The non-stationary character of the delocalized state results in a finite width $\Gamma$ of the 
scattering resonance as a function of the energy. The width and lifetime are related by the uncertainty relation $\tau \Gamma \simeq \hbar$. In the present problem the initial and finite states of the scattering process are the channel states of the lowest subband, the energy continuum starts above the transport threshold for the lowest subband, and the metastable states are localized within the ring.

The Fano interference [11] between the channel state carrying the current and the energy-degenerate localized state of a quantum ring or dot typically produces pronounced signatures in the electron transfer probability. The Fano resonances are extensively studied for quantum rings and open quantum dots side coupled to the channel [12, 13, 15, 14, 16, 17] as well as for potential cavities embedded within the channel [18, 19]. In order to determine the localized resonances we employ the stabilization method [20] in the version proposed by Mandelshtam and coworkers [21]. The method [21] determines the position and width of the resonances of the scattering probability by a bound-state calculations with square integrable eigenfunctions. In this approach one determines the energy spectrum of a finite system in function of its size. The spectrum contains energy levels that depend on the system size as well as size-independent energy levels. The size-dependent energy levels mimic the channel eigenstates and the size-independent energy levels correspond to wave functions that are localized at the scattering object. The latter are identified with the metastable states giving rise to the Fano interference [21].

In this paper we present - a first to our knowledge - study of the interplay of Fano resonances and magnetic forces in two- and three-terminal quantum rings at high magnetic field. For positive magnetic field the Lorentz force tends to inject the electron from the incoming lead to the left arm of the ring thus inducing a clockwise current circulation in the scattering eigenstate. At high magnetic field the Fano resonances involving localized states with clockwise orientation of persistent currents become too wide to be resolved in the transfer probability, while the resonances with anticlockwise currents become extremely sharp. We indicate that the changes in width of the resonances are consistent with the lifetime of ring localized states as obtained in a time-dependent simulation.

\section{Theory}

We study quasi two-dimensional structures in which the channels made of GaAs are embedded in $\mathrm{Al}_{x} \mathrm{Ga}_{1-x} \mathrm{As}$ matrix [22]. We consider three- and two- terminal open quantum rings of Fig. 1(a,b) and Fig. 2(b) as well as closed quantum rings with terminals of a finite length - Fig. 1(c) and Fig. 2(a). We consider systems in which the channels have $64 \mathrm{~nm}$ width [Fig. 1] that were addressed in the preceding work [10] as well as systems with channels that are only $32 \mathrm{~nm}$ wide [Fig. 2 in which the effect of magnetic deflection is weaker. We solve the Schroedinger equation

$$
H \Psi=E \Psi,
$$




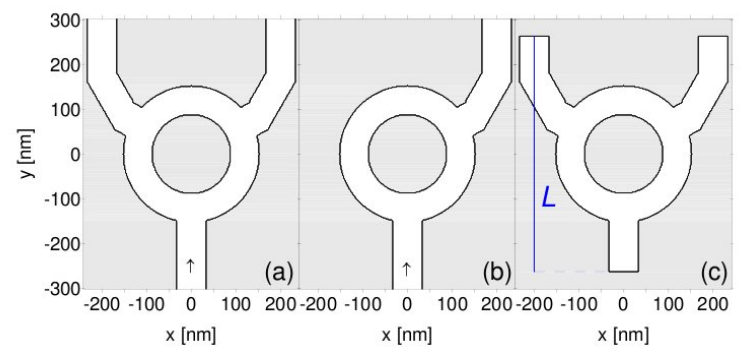

Figure 1. Open three terminal (a) and two terminal quantum rings (b) as well as their closed counterpart with terminals of finite width (c). The channels are $64 \mathrm{~nm}$ wide, the inner and outer radii of the ring are 88 and $154 \mathrm{~nm}$, respectively. The confinement potential is assumed zero within the white area and $200 \mathrm{meV}$ within the gray area. In (a) and (b) the lead with axis $x=0$ is treated as the input channel. $L$ in (c) denotes the length of the entire closed system.

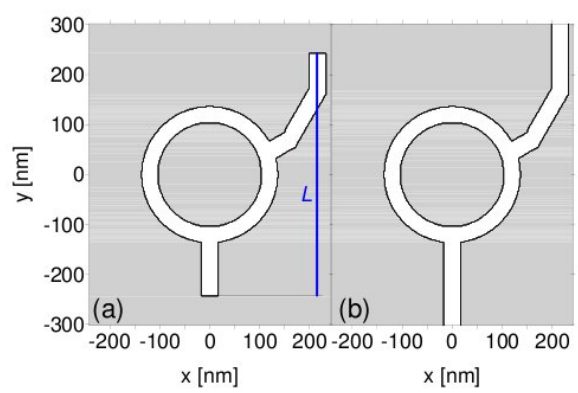

Figure 2. A closed quantum ring with two terminals of finite width (a) and an open two-terminal quantum ring (b) connected to infinite channels. The channels are $32 \mathrm{~nm}$ wide, the inner and outer radii of the ring are 104 and $136 \mathrm{~nm}$, respectively.

where

$$
H=(\mathbf{p}+e \mathbf{A}(\mathbf{r}))^{2} / 2 m^{*}+V(x, y),
$$

$m^{*}=0.067 m_{0}$ is the GaAs effective mass and the confinement potential $V$ is taken equal to zero inside the channels and $V_{0}=200 \mathrm{meV}$ outside. The adopted value of $V_{0}$ corresponds to the $\mathrm{Al}$ concentration in the matrix surrounding the channels of about $27 \%$. We assume that the magnetic field is oriented perpendicular to the plane of confinement and take the vector potential in the Landau gauge $\mathbf{A}=(0, B x, 0)$.

We are interested in the linear transport regime, which corresponds to a small bias $V$ applied between the terminals. The bias raises the Fermi energy of one of the terminals with respect to the other and allows for a flow of an uncompensated current through the system. The net linear current $J$ is proportional to the bias $V$, i.e. $J(B, V)=G(B) V$, where $G(B)$ is the conductance of the ring. In the LandauerButtiker approach the lowest-subband linear conductance is determined by the Fermi electron transfer probability from the input to the output lead $G(B)=\frac{2 e^{2}}{h} T(B)[23]$. The linear conductance does not depend on the bias. For that reason below we evaluate 
the transfer probability for $V=0$, which is a standard approach in the linear transport calculations. In our calculation the input lead is specified by the boundary condition and not by the bias. The current that we calculate and discuss below corresponds to the Fermi level electron coming from the input lead.

In the lowest-subband transport regime in each of the leads far away from the ring the energy levels are two-fold degenerate with one of the electron states going up and the other going down the channel. In order to evaluate the transfer probability $T$ we look for solution of eigenequation (1) for the electron that comes to the ring from the terminal that is attached to the ring from below [Fig. 1(a)]. In the output leads far from the ring one finds only the outgoing wave function

$$
\Psi(x, y)=a \exp (i q y) \psi^{q}(x),
$$

where $q$ is the wave vector and $\psi_{q}(x)$ is the transverse wave function [10]. In the input lead one finds both the incoming and outgoing wave functions

$$
\Psi(x, y)=b \exp (i k y) \psi^{k}(x)+c \exp (-i k y) \psi^{-k}(x) .
$$

The axis of the input lead is $x=0$, while the axes of the left and right output leads [Fig. 11 correspond to $x_{l}=-x_{r}=200 \mathrm{~nm}$. For fixed energy the wave vectors in the input and output leads are related as $q_{l}=k-\frac{e B}{\hbar} x_{l}$ and $q_{r}=k-\frac{e B}{\hbar} x_{r}$. The method of evaluation of the transfer probability was described in detail in Ref. [10]. We use the kinetic energy discretization of Ref. [24] that ensures independence of the finite-difference results of the chosen gauge. The scattering amplitudes $a, b$ and $c$ are determined in a self-consistent manner (see Ref. [10]).

In order to determine the energies of localized states we solve the algebraic problem obtained in the discretized version of the eigenequation (1) only with modified boundary conditions. We require the wave functions to vanish at the edges of computational box. We also assume that the terminals have a finite length - see Fig. 11(c) and Fig. 2(a), where $L$ is the length of the system. We determine the states localized within the ring using the stabilization method [21]. When $L$ is large enough the wave function of a localized state vanishes long before the end of the channel and the energy of the ringlocalized state no longer depends on $L$. Besides the states localized in the ring, the eigenequation is solved by wave functions in which the electron is localized inside the channels. The energies of these states decrease with $L$. The energy levels corresponding to states localized within the ring and the states of the channel enter into avoided crossing, so one cannot indicate any fixed value of $L$ which would guarantee separation of the ring-localized states from the channel states. Instead [21], the energy spectrum is calculated in function of $L$, and the energies of localized states are extracted by a detection counter defined as

$$
N(E)=\int d L \sum_{l} \delta\left(\left|E-E_{l}(L)\right| ; d E\right)
$$

where the summation over $l$ runs over eigenenergies of the closed system,

$$
\delta\left(\left|E-E_{l}(L)\right| ; d E\right)= \begin{cases}0 & \text { for }\left|E-E_{l}(L)\right| \geq d E \\ 1 & \text { for } \quad\left|E-E_{l}(L)\right|<d E\end{cases}
$$


and $d E$ is a small energy window. In the calculation we keep a constant mesh spacing of $2 \mathrm{~nm}$ and $L$ takes discrete values that are integer multiples of $4 \mathrm{~nm}$. We consider $L$ between 480 and $600 \mathrm{~nm}$. Under these assumptions Eq. (5) amounts in counting the energy levels that appear close to $E$ as $L$ is changed.

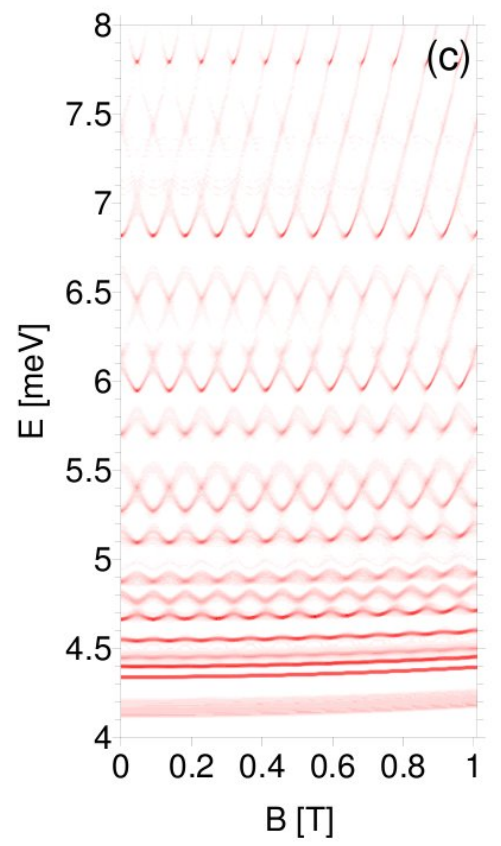

Figure 3. (a) Energy spectrum of a closed ring with two terminals attached [Fig. 2(a)] as a function of the length of the system for $B=0$. (b) The localized states detection counter $N$ [Eq. (5)] for $B=0$ obtained for the energy window $d E=10 \mu \mathrm{eV}$. (c) The resonance detection counter in function of $E$ and $B$. The darker the shade of red the larger value of $N$.

\section{Results and discussion}

\subsection{Thin channels}

For the clarity of the presentation it is useful to start from the ring with two terminals and thin channels of Fig. 2. We first extract the localized states by the stabilization method. We consider a closed system of a finite length $L$ that is depicted in Fig. 2(a). The eigenenergies found as function of $L$ are plotted in Fig. 3(a) for $B=0$. All the energies presented in Fig. 3(a) correspond to the energy continuum. For the considered channel width the continuum threshold is located at $3.89 \mathrm{meV}$ at $B=0(3.95 \mathrm{meV}$ at $B=1 \mathrm{~T})$. The energy levels that are independent of $L$ correspond to states with wave functions localized within the ring. The other energy levels correspond to delocalized states with wave functions that vanish only at the end of the channels. In Fig. 3(b) we displayed the localized states detection counter $N$ [Eq. (5)] calculated from the spectrum of Fig. 3(a) according to formula Eq. (5) with the energy window $d E=10 \mu \mathrm{eV}$. The 


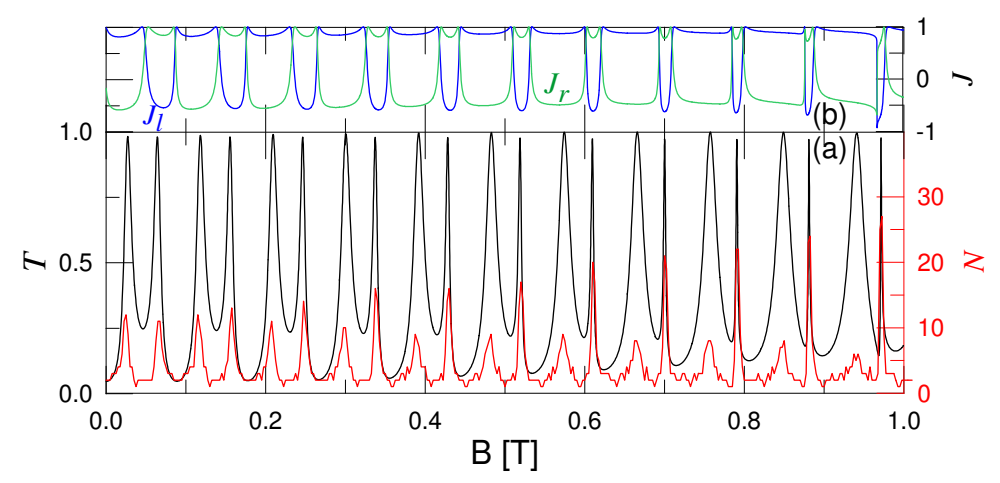

Figure 4. (a) The black curve shows the electron transfer probability (left axis) through the two terminal ring of Fig. 2 for the incident electron energy of $6 \mathrm{meV}$ and the red curve presents the localized resonance detection counter (right axis) - a cross section of Fig. 3(c). (b) The normalized density current fluxes through the left $J_{l}$ and right $J_{r}$ arms of the ring calculated for $y=0$ (see Fig. 2).

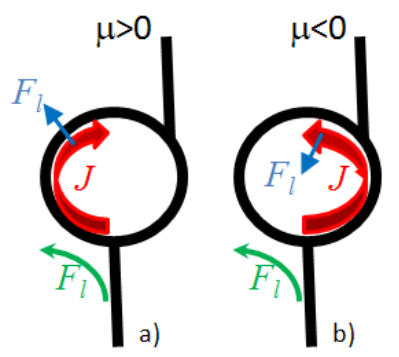

Figure 5. Schematic drawing of the probability current circulation $J$ (red arrows) in the resonant states corresponding to the positive (a) and negative (b) magnetic dipole moment $\mu$ at $B>0$. The green arrows indicate the Lorentz force that tends to inject the electron to the left arm of the ring. The blue arrows indicate the direction of the Lorentz force acting on the electron that circulates within the ring. For the circulation producing $\mu>0(\mu<0)$ the Lorentz force tends to eject the electron to the leads (keep the electron within the ring).

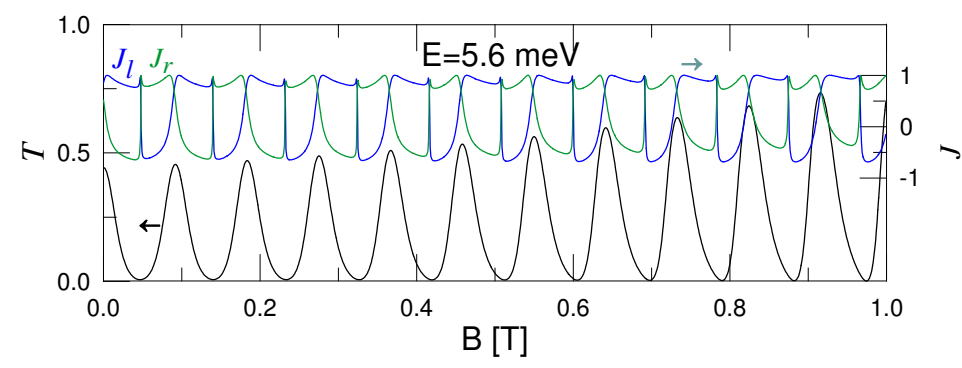

Figure 6. Same as Fig. 4 but for the incident electron of energy $5.6 \mathrm{meV}$. 
counter $N$ exhibits sharp peaks for energies for which a presence of a localized state is evident - see the flat energy level near $6.8 \mathrm{meV}$ in Fig. 3(a). $N$ forms wider maxima when a presence of localized state is less evident, in particular, where it is only suggested by a wider avoided crossings in Fig. 3(a) - see for instance the maximum of $N$ found near $6.1 \mathrm{meV}$.

In Fig. 3(c) we plotted how the maxima of $N$ move along the energy scale as the external magnetic field is applied. The plot exhibits a clear Aharonov-Bohm oscillation of the resonances with a period of about $0.091 \mathrm{~T}$, which corresponds to the magnetic field that produces a quantum of the flux $\Phi_{0}=h / e=B \pi R^{2}$ piercing a one-dimensional ring of an effective radius $R=120 \mathrm{~nm}$, which well agrees with the applied geometry (the arithmetic average of the inner and outer radii of the ring is $121 \mathrm{~nm}$ ). The resonant energy levels increase on average with $B$ due to the diamagnetic shift resulting from the finite width of the channels. For a closed perfectly circular quantum ring the angular momentum is a good quantum number and the energy levels cross. The avoided crossing that are present in Fig. 3(c) are due to angular momentum mixing by perturbation of the circular symmetry that is introduced by attachment of the input and output terminals [see Fig. 2].

In Fig. 4(a) we plotted with the red curve the values of $N$ found for $E=6 \mathrm{meV}$ in function of $B$. The result is obtained as a cross section of Fig. 3(c) for a fixed energy value. Within a magnetic field range of $0.091 \mathrm{~T}$ corresponding to a single flux quantum, we observe a double peak structure in the $N(B)$ dependence. As $B$ grows one of the peaks of the pair becomes sharper, and the other is transformed into lower and wider maximum. The sharp (wide) peaks of Fig. 4(a) correspond to localized energy levels that grow (decrease) in energy as $B$ grows [cf. Fig. 3(c)].

Let us now consider the open system [Fig. 2(b)] and an electron of energy $6 \mathrm{meV}$ that comes to the ring from below. The calculated transfer probability is displayed in Fig. 4(a) with the black line. We see that the transfer probability possesses peaks as functions of $B$ which coincide with the maxima of the resonance detection counter $N$. The result of Fig. 4(a) indicates that the transfer probability is governed by the interference of the channel state with a localized state, known as the Fano [11] interference. The transfer probability extrema are distinctly asymmetric which is a typical signature of this phenomenon [11].

Note, that in Fig. 4(a) a good agreement is obtained not only in the position but also in the width of $T$ and $N$ maxima. We find that the maxima of $T$ and $N$ become wider or thinner at higher $B$ depending on the direction of the persistent current circulation in the localized states. For a closed quantum ring the magnetic dipole moment generated by the persistent current flowing in the stationary state $\psi$ is defined as $\mu=-\frac{d E}{d B}=-\frac{d\langle\psi|H| \psi\rangle}{d B}$. For a strictly one-dimensional ring one obtains by an elementary algebra [25] a classical formula $\mu=-\frac{1}{2} e \mathbf{r} \times \mathbf{j}$, where $\mathbf{j}$ is the probability density current. The states whose energy increases (decreases) with growing $B$ produce the magnetic dipole moment which is antiparallel (parallel) to the external magnetic field with anticlockwise (clockwise) probability density current circulation around the 
ring (see Fig. 5).

In Fig. 4(b) we plotted the normalized probability density current fluxes calculated across the left and right arms of the ring across the line $y=0$. Note, that between adjacent maxima of $T$ and $N$ the circulation of the current changes orientation. The maxima of $T$ that become wider at high $B$ correspond to clockwise current circulation $\left(J_{r}<0, J_{l}>0\right.$, i.e. the current going down in the right arm and up in the left arm). The clockwise current circulation is consistent with the orientation of the current flow in the localized resonance that enters into degeneracy with the incident electron energy in the resonant range of the magnetic field. Moreover, the clockwise current agrees with the orientation of magnetic forces which tend to inject the electron from the input channel to the left arm of the ring (see the sketch of Fig. 5). The intervals of clockwise current circulation increase in width at higher $B$ and so do the corresponding Fano resonances of the transfer probability. The Fano resonances that become extremely thin at high $B$ involve localized states with anticlockwise current circulation that is opposite to the direction of the Lorentz force at the electron injection to the ring.

Fig. 4(b) contained a number of Fano peaks of the transfer probability obtained for the electron energy of $6 \mathrm{meV}$. Fig. 6 shows the transfer probability calculated for the incident electron energy of $5.6 \mathrm{meV}$, in the center of avoided crossings between the localized states [see Fig. 3(c)]. For this energy there are no conditions for Fano interference, i.e. at the energy of the incident electron no localized energy level is found for any magnetic field. Fig. 6 indicates that the transfer probability undergoes Aharonov-Bohm oscillation with no abrupt features present at high magnetic field. For the transfer through the localized states [Fig. 4] the peaks of $T$ corresponded to a distinct clockwise or anticlockwise current circulation. When the Fano interference is absent [Fig. 6] - at the peaks of $T$ - the current flows with the same intensity through both arms of the ring in spite of the asymmetry in the attachment of the leads to the ring [Fig. 2] accompanied by asymmetry of the magnetic forces. The effect of the magnetic injection is still visible in Fig. 6: the magnetic field windows that correspond to clockwise current circulation become wider at higher $B$ at the expense of these $B$ ranges in which the current flows anticlockwise.

\subsection{Wider channels}

Now let us turn our attention to the ring with wider channels [Fig. 1] in which the deflection of the electron trajectories by the Lorentz force is much stronger. The resonance detector counter as calculated for two [Fig. 11(b)] and three terminal [Fig. 1(b)] device is displayed in Figs. 7(a) and 7(b), respectively. The green curve in Fig. 7(a) displays the position of the continuum threshold, determined as the ground-state of an infinite channel that corresponds to zero wave vector. The two lowest energy lines correspond to bound states of which the ground-state is localized at the junction of the output terminal to the ring and the higher energy state - at the input terminal to the ring. Above the threshold one observes ring-localized resonant levels. As compared to 


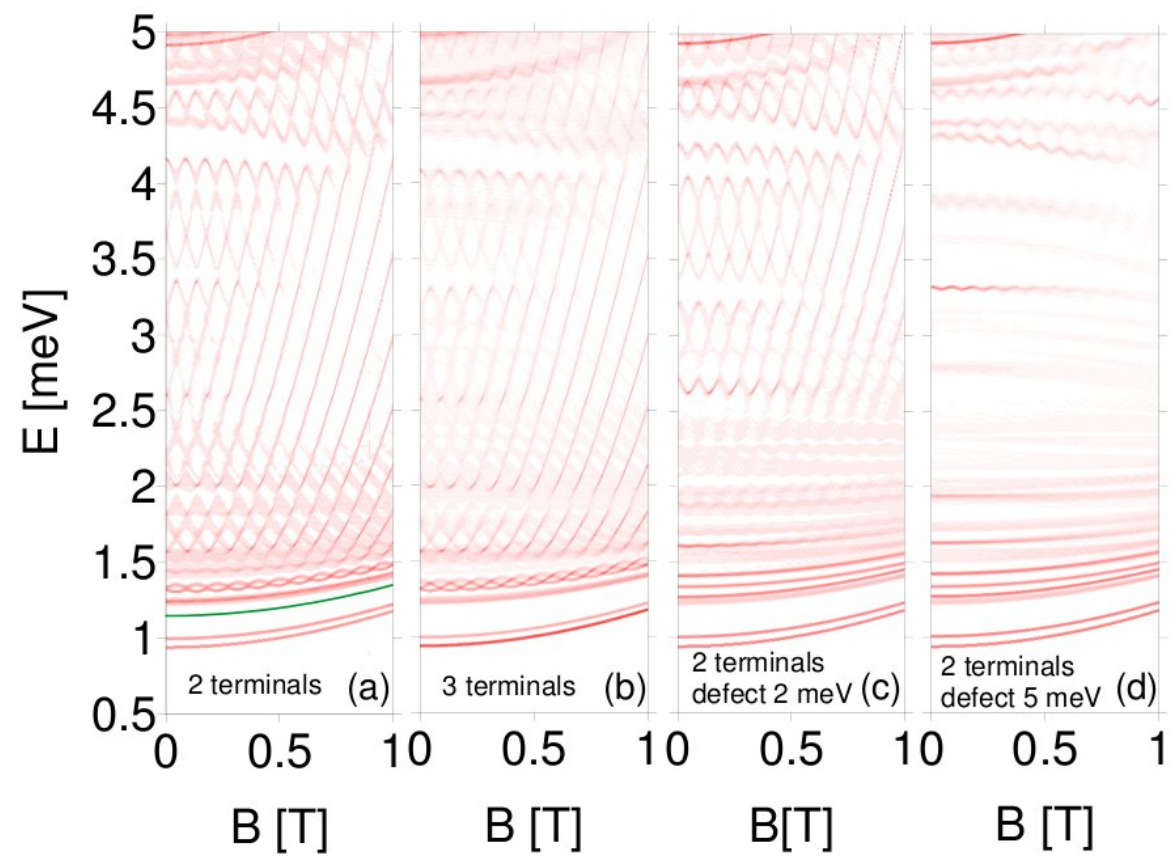

Figure 7. Resonance detection counter $N$ [see Eq. (5)] for two-terminal (a) and three-terminal (b) perfect ring. The darker the shade of red the larger the value of $N$. The results for a two-terminal ring with a repulsive Gaussian defect $(\mathrm{c}, \mathrm{d})$ present with the ring (see text). The height of the defect is taken equal to $2 \mathrm{meV}$ in (c) and $5 \mathrm{meV}$ in (d). In (a) by the green curve we plotted the energy of the continuum threshold.

the case with thinner channels presented in Fig. 3(c) the energy of the ring localized states is shifted down by about $3 \mathrm{meV}$ on the energy scale and the avoided crossings between the resonances are much thinner. The spectrum of resonant energy levels in two terminal [Fig. 7(a)] and three terminal [Fig. 7(b)] quantum rings are very similar.

In Fig. 8(b) we plotted the transfer probability (black line) and resonance detection counter (red line) for the incident electron energy of $3 \mathrm{meV}$. We can see that the double peak structure of $T$ evolves in the magnetic field much faster than for thinner channels [Fig. 4. In Fig. 8(a) we plotted the fluxes of the current through both arms of the ring. As previously [Fig. 4] the $T$ peaks with clockwise current circulation widen in higher $B$. The peaks of $T$ that are associated with current circulation that is anticlockwise - opposite to the magnetic injection - become very narrow at higher $B$. At higher $B$ the resonance detection counter resolves only the narrow Fano peaks. We can see that within a single $N$ maximum at higher $B$ one obtains a peak that is followed by an abrupt dip. With the exception of the abrupt dips the transfer probability becomes close to 1 and the oscillations acquire the Aharonov-Bohm periodicity with a decreasing oscillation amplitude.

The result for three terminal ring is displayed in Fig. 9. Each of the double peaks of $T=T_{l}+T_{r}$ correspond to transfer to the left $\left(T_{l}\right)$ or right $\left(T_{r}\right)$ output electrodes. $T$ for the three terminal ring is similar to the one obtained for two terminal structure 


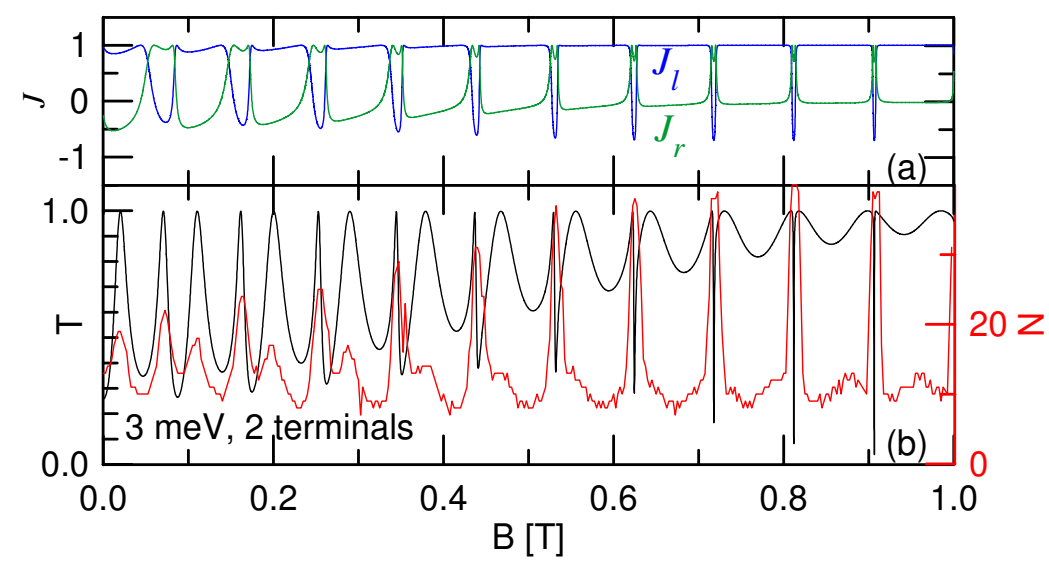

Figure 8. Electron transfer probability [black curve in (b) referred to the left axis] and resonance detection counter [red curve in (b) referred to the right axis] as functions of the magnetic field for a two terminal ring [see Fig. 1(b)]. The green and blue curves in (a) show the normalized current fluxes through the left and right arm of the ring calculated for $y=0$.

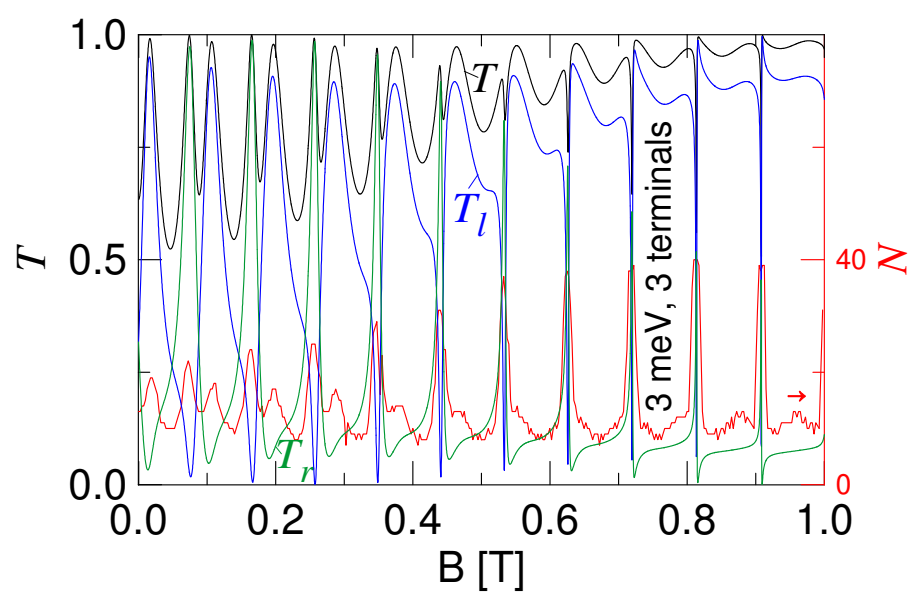

Figure 9. Electron transfer probability $\left[T=T_{l}+T_{r}\right.$, the black curve, the left axis] and resonance detection counter [the red curve, the right axis] as functions of the magnetic field for a three terminal ring [see Fig. 1(a)]. The green and blue curves in (a) show the transfer probability to the left and right output leads. The incident electron energy of $3 \mathrm{meV}$ is applied as in Fig. 8

[Fig. 8]. The dips of $T$ are associated with peaks of the transfer probability to the right 

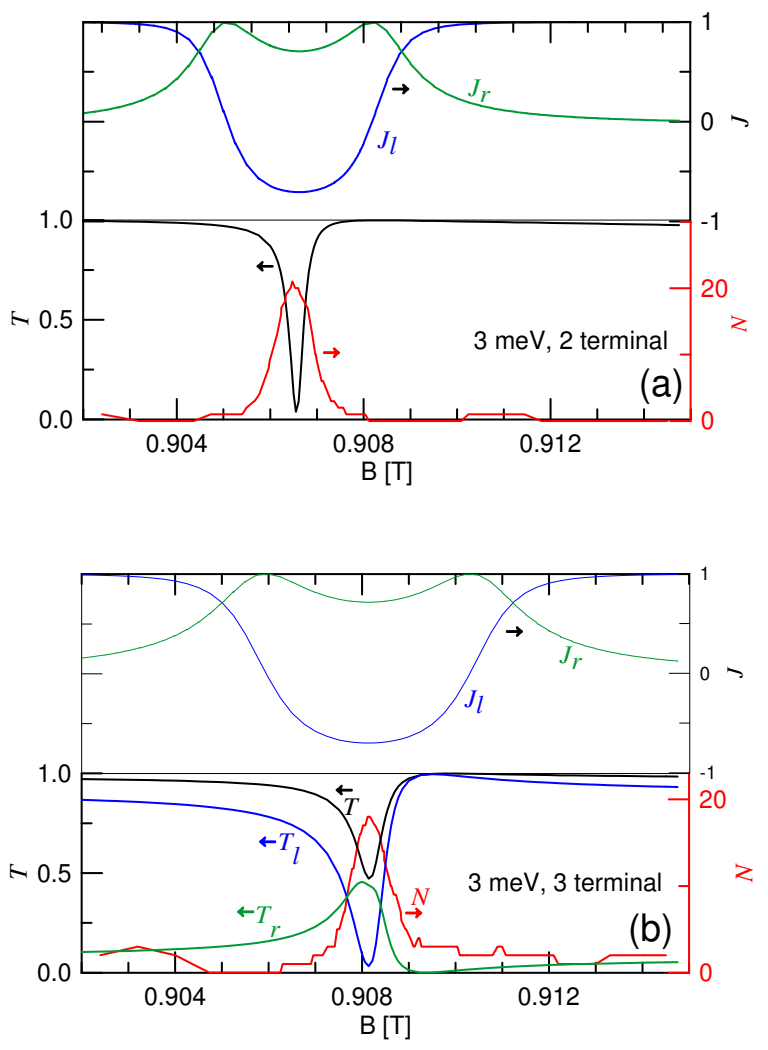

Figure 10. (a) and (b) show enlarged fragments of Fig. 8 and Fig. 9 respectively. In this plot the values of $N(B)$ were obtained for $d E=2 \mu \mathrm{eV}$.

output terminal $T_{r}$ and dips of $T_{l}$.

In Fig. 10 we plotted a zoom of the data that corresponds to the dip of $T$ near $0.91 \mathrm{~T}$ for both the two-terminal [Fig. 10(a)] and the three-terminal rings [Fig. 10(b)]. The dips of $T$ are distinctly asymmetric and coincide with the peak of $N$ as calculated for $d E=2 \mu \mathrm{eV}$. For three terminal ring [Fig. 10(b)] an asymmetric peak of $T_{r}$ coincides with an asymmetric dip of $T_{l}$.

\subsection{Lifetime of ring localized states versus the magnetic forces}

Figs. 3(a) and 7 indicate that at high magnetic field the resonances corresponding to localized states with $\mu<0(\mu>0)$ decrease (increase) in width as a function of both $B$ and $E$. The width of the Fano resonance as a function of the energy is related to the lifetime [11] of the corresponding localized state. In order to study the lifetime of ringlocalized states we solved the time-dependent Schroedinger equation with the method described in Ref. [10]. For the initial condition we took the eigenstates of a closed ring with no leads attached [see Figs. 11(b) and Figs. 11(e)] which in this simulation

play the role of the metastable states of the open system. We focused on the magnetic field near $0.91 \mathrm{~T}$ and on the electron energy of $3 \mathrm{meV}$ which was considered in the last 
subsection. The electron forms eigenstates of this energy at $0.905 \mathrm{~T}$ and $0.915 \mathrm{~T}$ in the closed ring [Fig. 11(a)]. The eigenstates possess a definite angular momentum of $-6 \hbar$ and $-17 \hbar$, respectively. The $l=-6$ state corresponds to $\mu<0$, the Lorentz force tends to keep the electron within the ring [Fig. 5(b)] and the charge density is pushed into the inner edge of the ring [Fig. 11(b)]. Opposite shift is observed for $l=-17$ which near $0.9 \mathrm{~T}$ corresponds to $\mu>0$, see Fig. 11(e).

In the initial moment of the simulation $(t=0)$ we attach the leads to the ring. For $l=-17$ the packet is promptly ejected to the channel [Fig. 11(f,g)], while for $l=-6$ the Lorentz force keeps the electron within the ring [Fig. 11(c,d)]. The part of the wave packet that is localized within the ring for both initial conditions are displayed in Fig. 12 with the red $(l=-17)$ and the black $(l=-6)$ lines. For $l=-6$ the ring-localized part falls to $50 \%$ near 100 ps as compared to 4.2 ps for $l=-17$.

For comparison we also performed simulations for eigenstates of the energy equal to $3 \mathrm{meV}$ at lower magnetic fields $\simeq 0.1 \mathrm{~T}$, where the Lorentz force is weaker. We took $l=6$ and $l=-8$ closed-ring eigenstates for the initial condition, for the corresponding magnetic fields of $0.0625 \mathrm{~T}$ and $0.12 \mathrm{~T}$. Fig. 12 shows that the decay of the probability to find the electron within the ring for these two initial states of opposite current circulation is similar.

\subsection{Rings with elastic scatterers}

The experimental data [9] for the three terminal quantum ring exhibit the conductance asymmetry to the two output leads at $B=0$, accompanied by a low visibility of conductance oscillations at low magnetic field. In Ref. [26] we demonstrated by wave packet simulations that both the 0T conductance asymmetry and low visibility of the oscillations can be explained by elastic scattering effects due to a repulsive defect present within the ring. No sharp features of the transfer probability that might be due to the Fano interference were observed in the experimental results [9]. A possible reason for their absence is the finite temperature effect since the sharp features due to the Fano resonances are thermally unstable [10]. However, in Ref. [10] (Fig. 14 in particular) we demonstrated that the presence of the elastic scatterer explaining the $0 \mathrm{~T}$ conductance asymmetry and low visibility of conductance oscillations implies removal of any sharp features of the transfer probability at high magnetic field also at $0 K$. The present study of the localized states explains this finding.

In order to establish the role of elastic scatterers for the localized resonances we considered the ring with wide channels and two terminals of Fig. 1(b). As an elastic scatterer we used a Gaussian potential defect [10, 26] $W(x, y)=V_{0} \exp \left(-\left(\left(x-x_{c}\right)^{2}+\right.\right.$ $\left.\left(y-y_{c}\right)^{2}\right) / R^{2}$ ) where the center of the defect $x_{c}, y_{c}$ is put in point $(-104,-60) \mathrm{nm}$ and its diameter $2 R=64 \mathrm{~nm}$ is taken equal to the width of the channel. The results for the localized states detection counter is displayed in Figs. 7(c) and 7(d) for the height of the defect of $2 \mathrm{meV}$ and $5 \mathrm{meV}$. In Fig. 7(c) we notice that the resonances of the energy lower than $2.5 \mathrm{meV}$ no longer exhibit Aharonov-Bohm periodicity. An 
electron with lower energy no longer tunnels across the defect and the circulation of the persistent current along the rings is significantly hampered. The confinement potential for these electron energies is of a bent quantum wire type rather than a quantum ring. In Fig. 7(d) we can see that the Aharonov-Bohm periodicity is removed of the energy spectrum below $4 \mathrm{meV}$. The resonances still exist, but their energies are nearly flat in function of $B$, and they only exhibit a diamagnetic shift. For a quantum ring without the defect [Fig. $7(a, b)]$ at a fixed value of the Fermi energy the resonances appear with the Aharonov-Bohm periodicity. When the elastic scatterer is present the resonances appear at a sweep of $B$ only for certain energies, and their appearance - if any - can only be occasional and not periodic in $B$, which explains the removal of sharp Fano resonances of the theoretical spectra [10] and is a likely reason for their absence also in the experimental data [9].

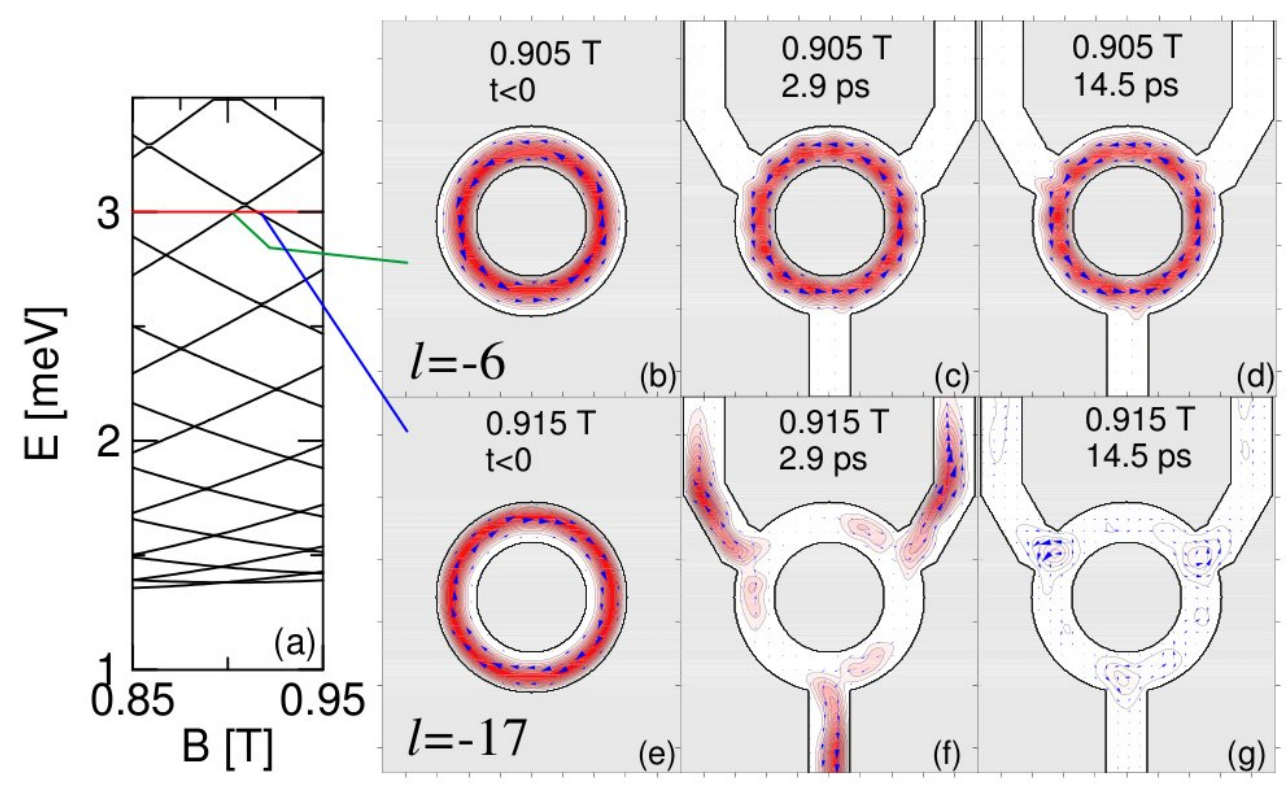

Figure 11. (a) Energy spectrum of a closed ring. (b-g) Snapshots of the timedependent simulations for the initial condition taken as the eigenstates of a closed ring of the energy of $3 \mathrm{meV}$, that for $B=0.905 \mathrm{~T}$ corresponds to angular momentum quantum number $l=-6$ (b) and for $B=0.915 \mathrm{~T}$ to $l=-17$ (e). (b) and (e) show the initial condition (eigenfunctions of a closed ring). The contours show the charge density and the blue arrows - the current density distribution. (c) and (d) show the results $2.9 \mathrm{ps}$ after the leads are attached to the ring. The snapshots corresponding to $t=14.5 \mathrm{ps}$ are displayed in (d) and (g).

\section{Summary and Conclusions}

We studied the electron transport through a two- and three-terminal quantum rings in external magnetic field. We demonstrated that at low magnetic field the electron transfer 


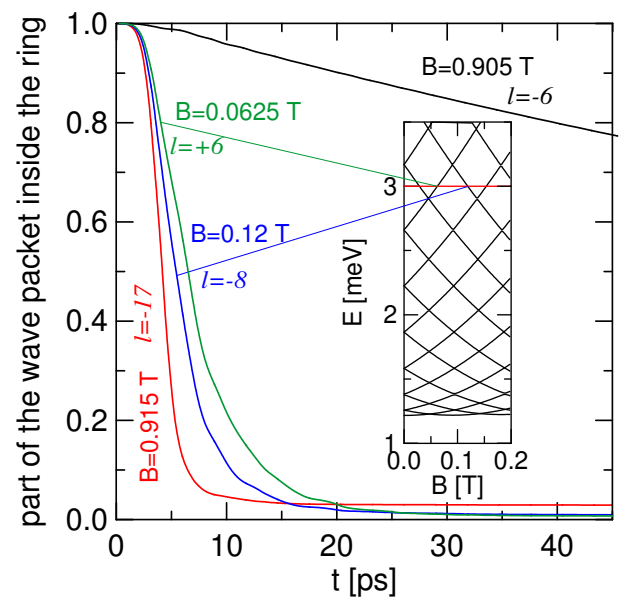

Figure 12. The red and black curves show the part of the wave packet localized within the ring for the time-dependent simulations presented in Fig. 11. The blue and green curves show the results of the simulation obtained for lower magnetic fields for $l=-8$ and $l=6$ angular momenta and the energy of $3 \mathrm{meV}$. Inset shows the closed ring spectrum for low magnetic fields.

probability bears signatures of Fano interference with localized states of both clockwise and anticlockwise persistent current orientation, which produce magnetic dipole moment that is parallel and antiparallel to the external magnetic field, respectively. In the localized states producing the magnetic dipole moment that is antiparallel to the magnetic field vector the Lorentz force tends to keep the electron within the ring thus enhancing the lifetime of the ring localized states. The corresponding resonances in the transfer probability dependence at high magnetic field become very narrow in function of both $B$ and the energy. Opposite is the behavior of Fano resonances involving localized states with current circulation which produces the magnetic dipole parallel to the external magnetic field: at high $B$ they become too wide to be resolved in the dependence of the transfer probability on the magnetic field. This current circulation (clockwise for $B>0$ ) is consistent with the direction of the magnetic-field-assisted electron injection to the ring (to the left arm of the ring for $B>0$ ). We also demonstrated that an elastic scatterer present within the ring destroys the Aharonov-Bohm periodicity of the Fano resonances.

Acknowledgements This work was performed within a research project N N202 103938 supported by Ministry of Science an Higher Education (MNiSW) for 2010-2013. Calculations were performed in ACK-CYFRONET-AGH on the RackServer Zeus.

[1] Y. Aharonov and D. Bohm, Phys. Rev. 115, 485 (1959).

[2] S. Olariu and I.I. Popescu, Rev. Mod. Phys. 57, 339 (1985).

[3] M. Büttiker, Phys. Rev. B 38, 9375 (1988).

[4] R. A. Webb, S. Washburn, C. P. Umbach, and R. B. Laibowitz, Phys. Rev. Lett. 54, 2696 (1985). 
[5] The Larmor radius is $R_{L}=m^{*} V_{F} / e B$ where $m^{*}$ is the effective mass and $V_{F}$ is the Fermi velocity. The semiconductor effective mass and the Fermi velocity for the two dimensional electron gas are much smaller than in metal. For Au $m^{*} \simeq m_{0}$ and the Fermi velocity is $1.4 \times 10^{6} \mathrm{~m} / \mathrm{s}$, which at $1 \mathrm{~T}$ gives the Larmor radius of about $R_{L}=8 \mu \mathrm{m}$, while of for a two-dimensional electron gas in GaAs $m^{*}=0.067 m_{0}$ with a typical density of $n=4 \times 10^{10} / \mathrm{cm}^{2}, E_{F}=\hbar \pi n / m^{*}$ the Fermi wave vector is $0.05 / \mathrm{nm}$ and $V_{F}=0.086 \times 10^{6} \mathrm{~m} /$, which produces $R_{L}=33 \mathrm{~nm}$.

[6] E. Strambini, L. Chirolli, V. Giovannetti, F. Taddei, R. Fazio, V. Piazza, and F. Beltram, Phys. Rev. Lett. 104, 170403 (2010); L. Chirolli, E. Strambini, V. Giovannetti, F. Taddei, V. Piazza, R. Fazio, F. Beltram, and G. Burkard, arXiv:1004.1895v1.

[7] T. Usuki, M. Takatsu, R. A. Kiehl, and N. Yokoyama, Phys. Rev. B 50, 7615 (1994).

[8] B. Szafran and F.M. Peeters, Europhys. Lett. 70, 810 (2005).

[9] E. Strambini, V. Piazza, G. Biasiol, L. Sorba, and F. Beltram, Phys. Rev. B 79, 195443 (2009).

[10] M. Poniedziałek and B. Szafran, J. Phys.: Condens. Matter 22, 21580 (2010).

[11] U. Fano, Phys. Rev. 124, 1866 (1961).

[12] A.A. Clerk, X. Waital, and P.W. Brouwer, Phys. Rev. Lett. 86, 4636 (2001).

[13] M.E. Torio, K. Hallberg, A.H. Ceccatto, and C.R. Proetto, Phys. Rev. B 65, 085302 (2002).

[14] T.F. Fang, W. Zuo, and J.Y. Chen, Phys. Rev. B 77, 125136 (2008).

[15] K. Kang, S.Y. Cho, J.J. Kim, and S.C. Shin, Phys. Rev. B 63, 113304 (2001).

[16] M.E. Torio, K. Hallberg, S. Flach, A.E. Miroshnichenko, and M. Titov, Eur. Phys. J. B 37, 399 (2004).

[17] M. Lee and C. Bruder, Phys. Rev. B 73, 085315 (2006).

[18] J. Göres, D. Goldhaber-Gordon, S. Heemeyer, M.A. Kastner, H. Shtrikman, D. Mahalu, and U. Meirav, Phys. Rev. B 62, 2188 (2000).

[19] C. Morfonios, D. Buchholz, and P. Schmelcher, Phys. Rev. B 80, 035301 (2009).

[20] A.U. Hazi and H.S. Taylor, Phys. Rev. A 1, 1109 (1970).

[21] V.A. Mandelshtam, T.R. Ravuri, and H.S. Taylor, Phys. Rev. Lett. 70, 1932 (1993).

[22] S. Pedersen, A. E. Hansen, A. Kristensen, C. B. Sørensen, and P. E. Lindelof Phys. Rev. B 61, 5457 (2000).

[23] S. Datta, Electronic Transport in Mesoscopic Systems (Cambridge Univ. Press, Cambridge, 1995).

[24] M. Governale and C. Ungarelli, Phys. Rev. B 58, 7816 (1998).

[25] B. Szafran, Phys. Rev. B 77, 205313 (2008).

[26] B. Szafran and M.R. Poniedziałek, Phys. Rev. B 80, 155334 (2009). 